\title{
Imprinted gene Zinc finger protein 127 is a novel regulator of master pluripotency transcription factor, Oct4
}

\author{
Yoo-Wook Kwon ${ }^{1, \#}$, Hyo-Suk Ahn ${ }^{2, \#}$, Joo-Young Park ${ }^{3}$, Han-Mo Yang ${ }^{4}$, Hyun-Jai Cho ${ }^{4}$ E Hyo-Soo Kim ${ }^{2,3,4,5, *}$ \\ ${ }^{1}$ Biomedical Research Institute, Seoul National University Hospital, Seoul 03080, ${ }^{2}$ Department of Molecular Medicine and \\ Biopharmaceutical Sciences, Graduate School of Convergence Science and Technology, Seoul National University, Seoul 03080, ${ }^{3}$ National \\ Research Laboratory for Stem Cell Niche, Seoul National University, Biomedical Research Institute, Seoul National University Hospital, \\ Seoul 03080, ${ }^{4}$ Department of Internal Medicine, Seoul National University Hospital, Seoul 03080, ${ }^{5}$ Strategic Center of Cell and Bio \\ Therapy for Heart, Diabetes \& Cancer, Seoul National University Hospital, Seoul 03080, Korea
}

Induced pluripotent stem cells (iPSCs) show great promise for replacing current stem cell therapies in the field of regenerative medicine. However, the original method for cellular reprogramming, involving four exogenous transcription factors, is characterized by low efficiency. Here, we focused on using epigenetic modifications to enhance the reprogramming efficiency. We hypothesized that there would be a new reprogramming factor involved in DNA demethylation, acting on the promoters of pluripotency-related genes. We screened proteins that bind to the methylated promoter of Oct4 and identified Zinc finger protein 127 (Zfp127), the functions of which have not yet been identified. We found that $\mathrm{Zfp} 127$ binds to the Oct4 promoter. Overexpression of Zfp127 in fibroblasts induced demethylation of the Oct4 promoter, thus enhancing Oct4 promoter activity and gene expression. These results demonstrate that Zfp127 is a novel regulator of Oct4, and may become a potent target to improve cellular reprogramming. [BMB Reports 2018; 51(5): 242-248]

\section{INTRODUCTION}

One of the major advantages of induced pluripotent stem cells (iPSCs) is their potential application in cell and regenerative therapies, and thus there have been numerous attempts to generate them. In 2006, a mouse fibroblast was successfully reprogrammed with four transcription factors, Oct4, Sox2, Klf4, and c-Myc (1). These transcription factors were ectopically

${ }^{*}$ Corresponding author. Tel: +82-2-2072-2226; Fax: +82-2-7668904; E-mail: hyosoo@snu.ac.kr

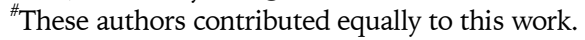

https://doi.org/10.5483/BMBRep.2018.51.5.196

Received 15 October 2017, Revised 11 November 2017, Accepted 11 January 2018

Keywords: Demethylation, Induced pluripotent stem cells, Oct4, Regenerative medicine, Zinc finger protein 127 overexpressed with viral transduction. Despite the many advantages that iPSCs can offer for cell therapy, low efficiency and tumorigenicity still remain major issues impeding their clinical applications. Therefore, many researchers have endeavored to improve their production efficiency, and to reduce the tumorigenic risks. Consequently, numerous experimental methods for generating iPSCs using plasmids, adenoviral transduction, miRNA, and protein extracts have been developed recently (2-7). Among them, several studies have focused on discovering new reprogramming factors (8-11).

It is widely known that gene expression patterns change with the epigenetic modifications made during reprogramming (12-15). The DNA of the promoters of pluripotency-related genes such as Oct4 and Nanog can change between methylated and unmethylated states (16-18). Oct4 mRNA is highly expressed in iPS cells, compared with somatic cells, which exhibit very low level expression. The differences in Oct4 expression originate from the methylation status of the Oct4 promoter. Therefore, we focused on the differences in the methylation patterns of the Oct 4 promoter, and attempted to identify a novel reprogramming factor associated with the demethylation of the Oct4 promoter.

Based on our previous studies $(6,19)$, where we generated iPSCs by exposing fibroblasts to cellular extract of embryonic stem cells or iPSCs, we hypothesized that the extract of pluripotent stem cells may contain reprogramming factors that bind to the methylated Oct4 promoter, and demethylate it during reprogramming. We aimed to discover these reprogramming factors by screening proteins bound to the methylated Oct4 promoter, and as a result, identified Zinc finger protein 127 (Zfp127). Although the function of Zfp127 has not been reported, its amino-acid sequence includes a zinc finger domain, leading us to predict that Zfp127 is capable of binding both DNA and RNA with high affinity. The Zfp127 gene is maternally imprinted, and located on mouse chromosome 7 (19).

Here, we have identified a novel function of Zfp127. We report that Zfp127 binds to the Oct4 promoter and

ISSN: 1976-670X (electronic edition)

Copyright (c) 2018 by the The Korean Society for Biochemistry and Molecular Biology

(c) This is an open-access article distributed under the terms of the Creative Commons Attribution Non-Commercial License (http://creativecommons.org/licenses/by-nc/4.0) which permits unrestricted non-commercial use, distribution, and reproduction in any medium, provided the original work is properly cited. 
demethylates it, leading to an increased expression of the Oct4 gene. We therefore speculate that Zfp127 influences the reprogramming process by positively regulating Oct4, the key transcription factor involved in reprogramming.

\section{RESULTS}

The Zfp127 gene was identified after screening proteins that bind to the methylated Oct4 promoter

CpG islands in the Oct4 promoter region are demethylated during cellular reprogramming, as shown in many previous studies $(1,2,6,20)$. Expression of Oct4 is largely regulated by the methylation status of the promoter. Therefore, we focused on finding the factor which binds to the methylated Oct4 promoter and modifies its methylation status. Methylation patterns on the Oct4 promoter show a distinct contrast between pluripotent stem cells and somatic cells $(21,22)$. We used the Oct4 promoter region, from -466 to +22 bp, relative to the transcription start site, in our experiments (Fig. 1A).

The Oct4 promoter was methylated artificially by CpG methyltransferase. Its methylation state was analyzed by digestion with $\mathrm{Mspl}$ and Hpall. Mspl recognizes the sequence, CCGG, digesting the Oct4 promoter into fragments of 266, 221 , and 46 base pairs. Hpall is an isoschizomer of Mspl, and is prevented from digesting at CCGG by the presence of a 5-methyl group at the internal $\mathrm{C}$ residue of its recognition sequence (Fig. 1B). Therefore, Mspl digested the methylated Oct4 promoter, but Hpall did not digest (Fig. 1C). Next, the methylated Oct4 promoter was incubated with protein extracts from mESC. Based on our previous studies where we generated iPSC by treating fibroblasts with mESC extracts, we hypothesized that the $\mathrm{mESC}$ extracts contain a reprogrammingrelated factor that binds to the Oct4 promoter $(6,7)$. To evaluate whether proteins were bound at Oct4 promoter with distinguishing methylation state, we performed western blot analysis with Oct4 and MBD2 antibodies after DNA (unmethylated or methylated Oct4 promoter) and proteins (mESC extract) were mixed together, then incubated and washed to remove unbound proteins. This process was described in Supplementary Fig. S1A as schematic figure. Oct4 protein was detected only at mixture of unmethylated DNA and mESC extract but MBD2 was detected at mixture of methylated DNA and $\mathrm{mESC}$ extract (Supplementary Fig. S1B). This is consistent with previous report (23). However, Lamin A/C, which contains no DNA binding domain, was detected neither mixture of methylated or unmethylated DNA and mESC extracts (data not shown). This data demonstrated that our screening system is valuable to find novel methylated DNA binding proteins. To discover methylated DNA binding proteins, DNA (methylated Oct4 promoter) and proteins (mESC extract) were mixed together, then incubated, washed and eluted the proteins, which were then separated by gel electrophoresis, purified, and analyzed by MALDI-TOF. We identified Zfp127 through this MALDI-TOF (Fig. 1D). We also

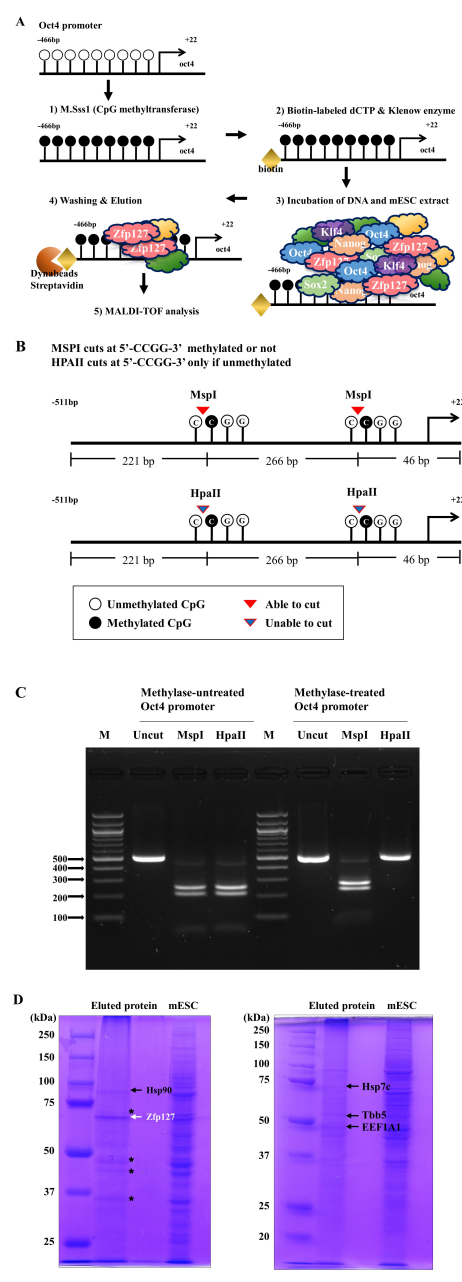

Fig. 1. Schematic diagram to screen proteins from the $m E S C$ extract that bind to the methylated Oct4 promoter. (A) Oct4 promoter (sequence from -466 to $+22 \mathrm{bp}$ ) was selected to screen the proteins from the mESC lysate that binds to the methylated Oct4 promoter. Artificially methylated Oct4 promoter was incubated with mESC extract. The proteins that bind to the methylated Oct4 promoter were eluted and separated using SDS-PAGE gel electrophoresis. They were then analyzed with MALDI-TOF. (B, C) The methylation state of the Oct4 promoter was confirmed by digestion with Msp I and Hpa II. (D) Eluted proteins included Hsp90, Hsp7c, Tbb5, EEF1A1 and Zfp127. *(asterisk) indicate unknown proteins.

identified Hsp90, Hsp7c, Tbb5, EEF1A1 through this analysis (Fig. 1D and Supplementary Table 1). Since the sequence of Zfp127 includes zinc finger domains, it can be translated into a putative protein with DNA or RNA binding ability. However, other proteins contain no DNA binding domain and are located at cytoplasm. Therefore, we speculated that only Zfp127 is likely to be a protein that binds to the methylated DNA. 


\section{Zfp127 increases Oct4 expression by inducing demethylation of the Oct4 promoter}

To investigate whether Zfp127 affects the gene expression of Oct4, we constructed a vector to overexpress the Zfp127 gene in the NIH-3T3 cell line and mouse embryonic fibroblasts (MEFs). The overexpression of Zfp127 was confirmed by mRNA and protein levels, using real-time PCR and western blot analysis respectively (Fig. 2A, B). Real-time PCR showed upregulated Oct4 mRNA expressions when Zfp127 was overexpressed. However, there was no change in gene expression levels of Nanog in the Zfp127 over-expressing cells (Fig. 2C). Next, we examined whether Zfp127 regulates the methylation state of the Oct4 promoter. After Zfp127 was overexpressed in NIH3T3 cells, we analyzed the methylation status of the Oct4 promoter using bisulfite sequencing. There are $16 \mathrm{CpG}$ islands in the Oct 4 promoter region from -466 to +20 bp. Among them, CpG islands located between +21 and $-244 \mathrm{bp}$ of the Oct4 promoter region were methylated, while the -268 to -466 bp region was completely unmethylated (Fig. 3A). The Oct4 promoter $(+21$ to -244 bp) region in the NIH-3T3 cell line had been reported as being unmethylated, which was thought to be a characteristic of this cell-line (24). Thus, we conducted bisulfite sequencing analysis in order to individually examine $10 \mathrm{CpG}$ islands within the promoter $(+21$ to $-244 \mathrm{bp})$ region of mock and Zfp127-transfected NIH3T3 cells. The frequency of the unmethylated CpG islands in Zfp127-transfected cells was twice as high as that of mock-transfected cells (Fig. 3B). The demethylation of the Oct 4 promoter can account for the increased Oct4 expression. This suggests that Zfp127 plays a role in demethylation of the Oct4 promoter and Oct4 gene expression, and may also facilitate cellular reprogramming. To rule out the possibility that Zfp127 can induce non-specific
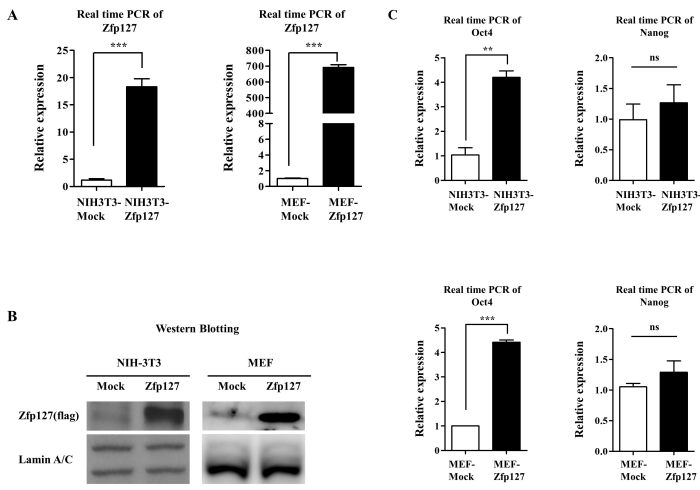

Fig. 2. Overexpression of Zfp127 increases Oct4 mRNA expression. (A, B) Real-time PCR and western blot results showed that high levels of Zfp127 were detected in Zfp127-overexpressing NIH3T3 cells and MEFs. (C) Overexpression of Zfp127 increased Oct4 expression, but not the expression of Nanog to significant levels. The asterisks indicate statistically significant changes: $* * \mathrm{P}$ $\leq 0.01, * * * P \leq 0.001$. demethylation of DNA, we analyzed the expression level of Nanog, and the methylation status of its promoter in Zfp127overexpressing cells. Unlike Oct4, Zfp127 overexpression did not affect the gene expression of Nanog (Fig. 2C), nor the methylation status of the Nanog promoter (Fig. 3C).

\section{Zfp127 binds to the Oct4 promoter and positively regulates its activity}

Next, we investigated whether the demethylation of the Oct4 promoter is linked to its activation. We constructed a luciferase vector containing a proximal enhancer and a promoter of Oct4 ( -466 to $+1 \mathrm{bp}$ ) (Fig. $4 \mathrm{~A}$ ), which was then co-transfected with the Zfp127 overexpression vector in $\mathrm{NIH} 3 \mathrm{~T} 3$ and MEFs. As shown in Fig. 4B, Zfp127 overexpression increased Oct4 promoter activity in NIH3T3 and MEFs by 1.7 and 2-fold greater, respectively, than the mock-control did. Next, to investigate whether Zfp127 binds and activates the Oct4 promoter, we performed a ChIP assay. The Zfp127 overexpression vector includes a FLAG tag, and thus we performed our ChIP assay with an anti-FLAG antibody. We confirmed that Zfp127 binds to the Oct4 promoter (Fig. 4C). This result demonstrates that the binding of Zfp127 increases the activity of Oct 4 promoter. Therefore, we can postulate that
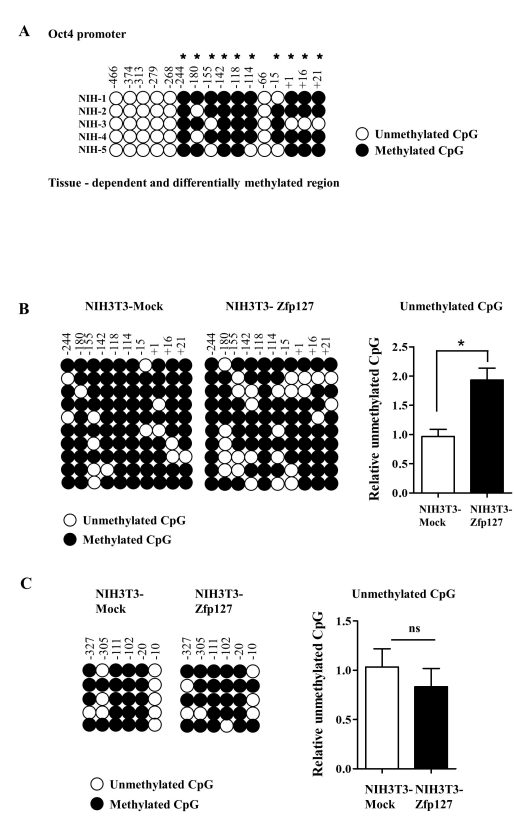

Fig. 3. DNA methylation analysis of Oct4 and Nanog promoters in Zfp127 overexpressing fibroblasts. (A) Methylation patterns of the $\mathrm{CpG}$ islands of the Oct4 promoter region from -466 to +21 bp in normal NIH-3T3 cells. Only $10 \mathrm{CpG}$ islands are methylated in this region. (B) Bisulfite sequencing analysis of 10 $\mathrm{CpG}$ islands of the Oct4 promoter. Partial demethylation of the Oct4 promoter was observed in the Zfp127-overexpressing $\mathrm{NIH}-3 \mathrm{~T} 3$ cells. (C) Demethylation of the Nanog promoter was not observed in the Zfp127 overexpressing NIH-3T3 cells. The asterisks indicate statistically significant changes: ${ }^{*} \mathrm{P} \leq 0.05$. 


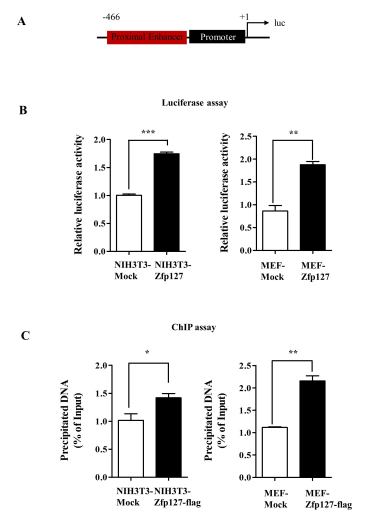

Fig. 4. Zfp127 activates and binds to the Oct4 promoter in luciferase assay. (A) The Oct4 promoter was cloned into the pGL2 luciferase vector. (B) Activation of the Oct4 promoter increased in Zfp127-overexpressing $\mathrm{NIH}$-3T3 cells and MEFs. (C) Quantification of the ChIP assay by real-time PCR with primers specific for the Oct4 promoter. The results are normalized to input. Binding of Zfp127 onto the Oct4 promoter increased in Zfp127 overexpressing NIH-3T3 cells and MEFs. The asterisks indicate statistically significant changes: $* P \leq 0.05, * * * P \leq 0.001$.

the induction of the Oct4 gene expression was due to the binding of Zfp127 on the Oct4 promoter, resulting in its demethylation. To confirm the transcriptional activity of Oct4, we investigated the mRNA levels of the well-known target genes of Oct4. Since Sox 2 and Foxd3 are early response genes of the Oct4 transcription factor (25), we choose them as downstream targets of Oct4. Real-time PCR showed upregulated mRNA levels of these genes when Zfp127 was overexpressed (Supplementary Fig. S2A). Combined, these results suggest that Oct4 is not only a direct target of Zfp127, but also has transcriptional activity.

\section{DISCUSSION}

Reprogramming somatic cells into pluripotent stem cells can be employed in various basic and clinical applications. Using iPSCs does not lead to problems such as ethical limitations and immune rejection after organ transplant. Despite many advantages of iPSCs in cell therapy, the low efficiency and time-consuming process of iPSC generation, as well as its tumorigenic risks, are significant handicaps for clinical applications. Through optimization of the reprogramming methods, these problems can be avoided (8-11, 26).

Our study aimed to find a new reprogramming-related protein, and to determine a novel function of this molecule, to contribute to the technological advancements in iPSC generation methods. Here, we designed a new experiment to screen reprogramming-related factors. Epigenetic changes occur when somatic cells are reprogrammed. One of the important epigenetic modifications which takes place during reprogramming is the removal of DNA methylation on the promoters of pluripotency-regulated genes $(16,21,22)$. We assumed that there would be some unknown reprogramming factors playing important roles in demethylating the Oct4 promoter. We hypothesized that mESCs bear these factors. This assumption is based on our previous study, for which we succeeded in making iPSCs by treating fibroblasts with protein extracts of mESCs $(6,7)$.

To screen for this DNA demethylase, we mixed and incubated the methylated Oct4 promoter with mESC extract, and analyzed the proteins that bound to the promoter, using MALDI-TOF (Fig. 1A). The methylation status of the Oct4 promoter is critical in regulation of Oct4 expression, as indicated by the different expression patterns seen in somatic and pluripotent cells $(18,21,22)$. For this reason, we chose to use the Oct4 promoter for our experiment. The analysis of our MALDI-TOF data allowed us to identify a complex of several compounds that bound to the methylated Oct4 promoter. The bands we obtained by SDS PAGE were a mixture of several different proteins, from which we were able to identify Hsp90, Hsp7c, Tbb5, EEF1A1, and Zfp127, as shown in the results (Fig. 1D and Supplementary Table 1).

Zfp127 is a maternally imprinted gene, expressed only by the paternal allele, and is translated into a putative protein possessing zinc finger motifs $(19,27)$. This imprinted region is homologous to human locus 15q11-q13, where its imprinting mutation results in Prader-Willi syndrome (PWS) (19). The mouse Zfp127 and the human orthologue show similar characteristics. They are expressed only by paternal alleles, and their sequences encode conserved proteins with five putative zinc finger motifs. These motifs imply that this protein may play important roles in DNA or RNA binding. The in vitro translation of human Zfp127 showed that it is likely to be translated in vivo and may encode a functional protein (27). This suggests that mouse Zfp127 may also encode a functional protein, and it is likely to regulate gene expression through its zinc finger motifs.

Although several proteins were obtained during the experiment, Zfp127 was selected as a feasible reprogramming factor based on its zinc finger motifs. The function of the $Z f p 127$ gene remains to be unveiled. Hence, we investigated the effects of the Zfp127 gene on the Oct4 promoter. As mentioned above, Zfp127 may affect the Oct4 promoter since expression of Oct4 mRNA increased in Zfp127 overexpressing cells. For this reason, we postulated that it may be due to the effects of Zfp127 on the Oct4 promoter.

We examined the methylation status of the Oct4 promoter and found that methylation of some CpGs were removed in the Zfp127 overexpressing cells. Next, to find out whether Zfp127 can demethylate other regions of the promoters of pluripotent factors nonspecifically, we checked the expression of the Nanog gene and its promoter methylation status, as Nanog is also a key transcription factor involved in reprogramming. However, neither the removal of the $\mathrm{CpG}$ methylations, nor the change in gene expression of Nanog 
were observed. Next, we confirmed the activation of the Oct4 promoter with a luciferase assay. Our analysis of the Oct4 promoter using bisulfite sequencing and a luciferase assay leads us to believe that the activation of the Oct4 promoter might be due to its demethylation.

In this study, we have demonstrated that Zfp127 induces Oct4 expression by demethylation of the Oct4 promoter specifically. However, Zfp127 contains no demethylase domain. Whether other DNA demethylating proteins are recruited to the proximal region, followed by Zfp127, remains to be investigated. The fact that the Zfp127 protein possesses zinc finger motifs and induces demethylation of $\mathrm{CpG}$ islands suggests that Zfp127 may have the ability to bind to the promoters of reprogramming-related genes other than Nanog. It is possible that there may be some other factors that interact with Zfp127, which influence the specificity of the binding sites.

To identify proteins that have DNA demethylase domains and interact with Zfp127 in mESC extracts, we performed a proteomic analysis using iTRAQ and Mass/Mass experiment. We compared pull-downs of mESC proteins, which were bound to either unmethylated or methylated Oct4 promoters respectively. However, the analysis did not identify any known DNA demethylation-related proteins, such as DNA deaminase (18) or methylcytosine oxidases (28). This suggests that there are unknown DNA demethylases which interact with Zfp 127 to demethylate the Oct4 promoter.

The finding that Zfp127 increases Oct4 expression, may be useful for practical applications, such as facilitating cellular reprogramming and improving the efficiency of generating iPSCs, offering the possibility of Zfp127 as a new reprogramming factor. The combination of Zfp127 and other known transcription factors can be introduced to somatic cells to enhance reprogramming efficiency.

In summary, we have identified a novel function of an imprinting gene, Zfp127. It binds to the Oct4 promoter, leading to demethylation of the Oct4 promoter. The promoter of Oct 4 was then activated, and the expression of Oct 4 mRNA increased. Therefore, we speculate that Zfp127 offers the potential for somatic cells to be reprogrammed into iPSCs with high efficiency.

\section{MATERIALS AND METHODS}

\section{Cell culture}

Mouse embryonic stem cells were derived from C57BL/6background mouse embryonic stem cells (American Type Culture Collection). mESCs were cultured as described in previously $(6,20)$. Briefly, one day before sub-culturing mESCs, mitomycin C $(10 \mu \mathrm{g} / \mathrm{ml}$ medium (M4287)(SigmaAldrich, USA))-treated STO cells were seeded on a new $0.1 \%$ gelatin-coated dish. The STO cells were cultured in Dulbecco's modified Eagle's medium (DMEM)(11995-065) (Invitrogen, USA) high glucose, supplemented with $10 \%$ Fetal
Bovine Serum (FBS)(16000-044)(Invitrogen, USA), and 100 $\mathrm{U} / \mathrm{ml}$ penicillin streptomycin (Gibco). $\mathrm{mESC}$ were cultured in (DMEM)(11995-065)(Invitrogen, USA) with $10 \% \quad$ FBS (FBS)(16000-044)(Invitrogen, USA), 2 mM L-glutamine (25030081)(Invitrogen, USA), 1X NEAA (Gibco) (11140-050) (Invitrogen, USA), $1 \mathrm{mM}$ 2- mercaptoethanol (Sigma-Aldrich), and $100 \mathrm{U} / \mathrm{ml}$ penicillin $/ 100 \mu \mathrm{g} / \mathrm{ml}$ streptomycin (Gibco) (15140-122)(Invitrogen, USA)(ES media). In ES media, 2,000 $\mathrm{U} / \mathrm{ml}$ of $\mathrm{ESGRO}^{\mathbb{R}}$ LIF (leukemia inhibitory factor; (ESG1107) (Chemicon, USA)) was added to maintain pluripotency. mESC were dissociated with $0.05 \%$ trypsin (Gibco)(25300-054) (Invitrogen, USA), and passaged on STO every 2-3 days. Cells from passages 5 to 7 were used for further experiments.

The NIH-3T3 cell line and the mouse embryonic fibroblasts were purchased from the American Type Culture Collection. $\mathrm{NIH}-3 \mathrm{~T} 3$ and MEFs were incubated at $37^{\circ} \mathrm{C}$ in Dulbecco's modified Eagle's medium (DMEM)(11995-065)(Invitrogen, USA), high glucose, supplemented containing 10\% Fetal Bovine Serum (FBS)(16000-044)(Invitrogen, USA), and 100 $\mathrm{U} / \mathrm{ml}$ penicillin streptomycin (Gibco)(15140-122)(Invitrogen, USA).

\section{Extraction of $\mathrm{mESC}$ protein}

mESCs were washed in PBS and harvested with $0.1 \mathrm{mM}$ trypsin/1 mM EDTA solution, as described previously $(6,29)$. Cells were collected by centrifugation (200 g for $10 \mathrm{~min}$ at $4^{\circ} \mathrm{C}$ ) and resuspended in $1 \mathrm{ml}$ cold cell lysis buffer (100 mM HEPES, pH 8.2, $50 \mathrm{mM} \mathrm{NaCl}, 5 \mathrm{mM} \mathrm{MgCl} 2,1 \mathrm{mM}$ dithiothreitol, and protease inhibitor cocktail (04 693132 001)(Roche, Switzerland). Cells were mixed by vortexing on ice for $1 \mathrm{~h}$, and homogenized using a 20-gauge needle. The lysate was centrifuged at $15,000 \mathrm{rpm}(22,250 \mathrm{~g})$ for $30 \mathrm{~min}$ at $4^{\circ} \mathrm{C}$, and the supernatant was stored at $-80^{\circ} \mathrm{C}$. The protein concentration was measured with a BCA protein assay kit (23255)(Thermo Scientific, USA).

\section{In vitro methylation assay}

An amplified Oct4 promoter product (500 ng) was methylated by M.Sssl methylase (M0226S)(New England Biolabs, UK), at $37^{\circ} \mathrm{C}$ for $3 \mathrm{~h}$, with the subsequent inactivation of enzyme at $60^{\circ} \mathrm{C}$ for $20 \mathrm{~min}$. To identify of the methylation state of Oct 4 promoter, the methylated product was digested with Msp I (R057S)(Enzynomics, Korea) and Hpa II (R049S)(Enzynomics, Korea).

Screening for the methylated Oct4 promoter binding protein A vector containing mouse Oct4 promoter was constructed. The DNA vector was treated with the appropriate restriction enzyme, and only the mouse Oct4 promoter was purified. The purified DNA was methylated artificially with CpG Methyltransferase (M.Sssl;(M0226S)(New England Biolabs, UK)), and biotinylated with biotin-labeled dCTP and Klenow enzyme. Biotinylated DNA was attached to streptavidin with Dynabeads kilobaseBINDER kit (60101)(Invitrogen, USA). The 
DNA was then incubated with the mESC protein extract. To remove the unbound proteins, beads were washed with washing buffer and magnets. Magnets pull streptavidin-Dynabeads, which were attached to biotinylated DNA with bound proteins. The last step was the elution of the proteins from the DNA and the protein analysis. After treatment with DNase (M6101)(Promega, USA) to eliminate DNA, we carefully took the supernatant. Finally, eluted proteins were separated using SDS-PAGE gel electrophoresis, and analyzed with MALDI-TOF and western blotting with Oct4 (sc-5279)(Santa Cruz, USA) and MBD2 (ab38646) (Abcam, UK) antibodies.

\section{Western blot analysis}

Cells were harvested and lysed in lysis buffer containing protease inhibitor cocktail (04 693132 001)(Roche, Switzerland). Total protein (20-30 mg) was immunoblotted with primary antibodies against FLAG (F7425)(Sigma Aldrich, USA). Lamin A/C (2032S)(Cell Signaling Technology, USA), was used as an internal control.

\section{Plasmid construction and transfection}

The Zfp127 gene was amplified with 2x Pfu premix (Solgent). RNA of $\mathrm{mESC}$ was used as a template. The amplified DNA was ligated into the T-Blunt vector using a T-Blunt PCR Cloning Kit (SOT02-K020)(Solgent, Korea). The Zfp127 gene was sub-cloned into FLAG tagged pCMV-Tag 2B (FLAG-Zfp127). The whole sequence of $Z f p 127$ was confirmed through DNA sequencing. To overexpress Zfp127, FLAG-Zfp127 was transfected to NIH-3T3 cells by using of polyethyleneimine (PEI (23966)(polyscience, Inc. USA)) and was transfected to MEFs, by using the Amaxa nucleofection system (Lonza, Switzerland).

\section{RNA preparation and PCR analysis}

The total RNA of each cell line was purified with RNeasy Mini Kit (74104)(Qiagen, Germany), and cDNA was synthesized with an amfiRivert cDNA synthesis premix (Gendepot). The conventional RT-PCR was performed with a Maxime PCR Premix (25265)(Intron Biotechnology, Korea), and quantitative real-time PCR was performed with SYBR Green master mix (04913914001)(Roche, Switzerland). The following primer sets were used: 5'-AAGCGTGCAGGTATTTTGCT-3' and 5'ACTGCACAGGC TCCAAAACT-3' for the Zfp 127 gene; 5'GAGGAGTCCCAG GACATGAA-3' and 5'-AGATGGTGGTCT GGCTGAAC-3' for the Oct4 gene.

Genomic DNA preparation and bisulfite sequencing analysis Genomic DNA of each cell line was extracted with a Genomic DNA extraction kit (17045)(Intron Biotechnology, korea). Conversion of the genomic DNA was performed with an EpiTect Bisulfite kit (59104)(Qiagen, Germany). The converted
DNA was used as a template to amplify the Oct4 promoter region. Amplified DNA was then purified and ligated to the pGEM-T easy vector (A1360)(Promega, USA) for sequencing. The primer sets used for the bisulfite sequencing are as follows:

5'-TGGGTTGAAATATTGGGTTTATTT-3'and 5'-CTAAA AC CAAATATCCAACCATA-3' for the Oct4 gene, 5'-GATTTGTAG GTGGGATTAATTGTGAATTT-3'and 5'-ACCAAAAA AACCCA CACTC ATATCAATATA-3' for the Nanog gene (30).

\section{Luciferase assay}

To study Oct4 promoter activity, cells were transfected with pGL2-Oct4 promoter, luciferase reporter vector, and Renilla vector using polyethyleneimine (PEI)(23966)(polyscience, Inc. USA), approximately 1 or 2 days before detection. Cells were lysed with lysis buffer, and luciferase activity was detected with Dual-Luciferase Reporter Assay System (E1910)(Promega, USA).

\section{Chromatin immunoprecipitation assay}

A chromatin immunoprecipitation (ChIP) assay was performed as described previously $(19,28)$. In brief, Zfp127 overexpression was performed in $\mathrm{NIH}-3 \mathrm{~T} 3$ and MEFs as mentioned above. $1 \times 10^{6}$ cells of Zfp127-overexpressing NIH-3T3 and MEFs were rinsed and treated with $1 \%$ formaldehyde for 20 $\mathrm{min}$ at room temperature. Samples were then sonicated on ice and incubated with the antibodies; anti-FLAG (sc-807)(Santa Cruz, USA) and anti-IgG antibody at $4^{\circ} \mathrm{C}$ overnight. Immunoprecipitated DNA was analyzed using real-time PCR. The following primers were used to amplify the primer of the Oct4 promoter; forward,

5'-GGAGGTGCAATGGCTGTCTTGTCC-3', and reverse, 5'CTGCCTTGGGTCACCTTACACCTCAC-3'.

\section{Statistical analysis}

The results are expressed as means \pm standard deviations (SD). The differences between the groups were compared using an unpaired t-test, followed by post-hoc analysis with the Bonferroni test, and $\mathrm{P}$ values $\leq 0.05$ were considered statistically significant. All statistical analyses were performed using SPSS 17.0 (SPSS Inc., USA).

\section{ACKNOWLEDGEMENTS}

This study was supported by the Korea Health Technology R\&D Project (HI-17 C-2085 \& HI-14 C-1277) through the Korea Health Industry Development Institute (KHIDI) funded by the Ministry of Health \& Welfare (MHW).

\section{CONFLICTS OF INTEREST}

The authors have no conflicting interests. 


\section{REFERENCES}

1. Takahashi K and Yamanaka S (2006) Induction of pluripotent stem cells from mouse embryonic and adult fibroblast cultures by defined factors. Cell 126, 663-676

2. Okita $K$, Nakagawa $M$, Hyenjong $H$, Ichisaka $T$ and Yamanaka S (2008) Generation of mouse induced pluripotent stem cells without viral vectors. Science 322, 949-953

3. Zhou W and Freed CR (2009) Adenoviral gene delivery can reprogram human fibroblasts to induced pluripotent stem cells. Stem Cells 27, 2667-2674

4. Miyoshi N, Ishii H, Nagano H et al (2011) Reprogramming of mouse and human cells to pluripotency using mature microRNAs. Cell Stem Cell 8, 633-638

5. Xu N, Papagiannakopoulos T, Pan G, Thomson JA and Kosik KS (2009) MicroRNA-145 regulates OCT4, SOX2, and KLF4 and represses pluripotency in human embryonic stem cells. Cell 137, 647-658

6. Cho HJ, Lee CS, Kwon YW et al (2010) Induction of pluripotent stem cells from adult somatic cells by protein-based reprogramming without genetic manipulation. Blood 116, 386-395

7. Kim D, Kim CH, Moon Jl et al (2009) Generation of human induced pluripotent stem cells by direct delivery of reprogramming proteins. Cell Stem Cell 4, 472-476

8. Yu J, Vodyanik MA, Smuga-Otto K et al (2007) Induced pluripotent stem cell lines derived from human somatic cells. Science 318, 1917-1920

9. Sridharan R, Tchieu J, Mason MJ et al (2009) Role of the murine reprogramming factors in the induction of pluripotency. Cell 136, 364-377

10. Zhao Y, Yin X, Qin $\mathrm{H}$ et al (2008) Two supporting factors greatly improve the efficiency of human iPSC generation. Cell Stem Cell 3, 475-479

11. Silva J, Nichols J, Theunissen TW et al (2009) Nanog is the gateway to the pluripotent ground state. Cell 138, $722-737$

12. Hanna J, Saha K, Pando B et al (2009) Direct cell reprogramming is a stochastic process amenable to acceleration. Nature 462, 595-601

13. Buganim Y, Faddah DA, Cheng AW et al (2012) Single-cell expression analyses during cellular reprogramming reveal an early stochastic and a late hierarchic phase. Cell 150, 1209-1222

14. Eminli S, Foudi A, Stadtfeld M et al (2009) Differentiation stage determines potential of hematopoietic cells for reprogramming into induced pluripotent stem cells. Nat Genet 41, 968-976

15. Shin J and Youn HD (2016) Oct4 resetting by Aurkb-PP1 cell cycle axis determines the identity of mouse embryonic stem cells. BMB Rep 49, 527-528

16. Freberg CT, Dahl JA, Timoskainen S and Collas P (2007) Epigenetic reprogramming of OCT4 and NANOG regulatory regions by embryonal carcinoma cell extract.
Mol Biol Cell 18, 1543-1553

17. Hochedlinger K and Plath K (2009) Epigenetic reprogramming and induced pluripotency. Development 136, 509-523

18. Bhutani N, Brady JJ, Damian M, Sacco A, Corbel SY and Blau HM (2010) Reprogramming towards pluripotency requires AID-dependent DNA demethylation. Nature 463, 1042-1047

19. Jong MT, Carey AH, Caldwell KA et al (1999) Imprinting of a RING zinc-finger encoding gene in the mouse chromosome region homologous to the Prader-Willi syndrome genetic region. Hum Mol Genet 8, 795-803

20. Kwon YW, Paek JS, Cho HJ et al (2015) Role of Zscan4 in secondary murine iPSC derivation mediated by protein extracts of ESC or iPSC. Biomaterials 59, 102-115

21. Imamura $M$, Miura $K$, Iwabuchi $K$ et al (2006) Transcriptional repression and DNA hypermethylation of a small set of ES cell marker genes in male germline stem cells. BMC Dev Biol 6, 34

22. Okita K, Ichisaka T and Yamanaka S (2007) Generation of germline-competent induced pluripotent stem cells. Nature 448, 313-317

23. Gu P, Xu X, Le Menuet D, Chung AC and Cooney AJ (2011) Differential recruitment of methyl CpG-binding domain factors and DNA methyltransferases by the orphan receptor germ cell nuclear factor initiates the repression and silencing of Oct4. Stem Cells 29, 1041-1051

24. Jin SG, Guo C and Pfeifer GP (2008) GADD45A does not promote DNA demethylation. PLoS Genet 4, e1000013

25. Sharov AA, Masui S, Sharova LV et al (2008) Identification of Pou5f1, Sox2, and Nanog downstream target genes with statistical confidence by applying a novel algorithm to time course microarray and genome-wide chromatin immunoprecipitation data. BMC Genomics 9, 269

26. Lee JE, Chung YG, Eum JH, Lee Y and Lee DR (2016) An efficient SCNT technology for the establishment of personalized and public human pluripotent stem cell banks. BMB Rep 49, 197-198

27. Jong MT, Gray TA, Ji Y et al (1999) A novel imprinted gene, encoding a RING zinc-finger protein, and overlapping antisense transcript in the Prader-Willi syndrome critical region. Hum Mol Genet 8, 783-793

28. Hill PW, Amouroux R and Hajkova P (2014) DNA demethylation, Tet proteins and 5-hydroxymethylcytosine in epigenetic reprogramming: an emerging complex story. Genomics 104, 324-333

29. Cha MY, Kwon YW, Ahn HS et al (2016) Protein-induced pluripotent stem cells ameliorate cognitive dysfunction and Reduce Abeta deposition in a mouse model of alzheimer's disease. Stem Cells Transl Med 6, 293-305

30. Hattori N, Nishino K, Ko YG et al (2004) Epigenetic control of mouse Oct-4 gene expression in embryonic stem cells and trophoblast stem cells. J Biol Chem 279, 17063-17069 